\title{
Особенности спектров фотовозбуждения эпитаксиальных слоев $\ln N$, выращенных методом молекулярно-пучковой эпитаксии с плазменной активацией азота
}

\author{
(ㄱ П.А. Бушуйкин ${ }^{1}$, А.В. Новиков ${ }^{1,2}$, Б.А. Андреев ${ }^{1,2}$, Д.Н. Лобанов ${ }^{1,2,9}$, П.А. Юнин ${ }^{1}$, \\ Е.В. Скороходов ${ }^{1}$, Л.В. Красильникова ${ }^{1,2}$, Е.В. Демидов ${ }^{1}$, Г.М. Савченко ${ }^{3}$, В.Ю. Давыдов ${ }^{3}$ \\ ${ }^{1}$ Институт ффизики микроструктур Российской академии наук, \\ 603950 Нижний Новгород, Россия \\ ${ }^{2}$ Нижегородский государственный университет им. Н.И. Лобачевского, \\ 603950 Нижний Новгород, Россия \\ ${ }^{3}$ Физико-технический институт им. А.Ф. Иофрфе Российской академии наук, \\ 194021 Санкт-Петербург, Россия \\ ฯ E-mail: dima@ipmras.ru
}

(Получена 23 апреля 2017 г. Принята к печати 12 мая 2017 г.)

\begin{abstract}
Представлены результаты исследования спектров фотовозбуждения эпитаксиальных слоев InN c концентрацией свободных носителей $10^{18}-10^{19} \mathrm{~cm}^{-3}$, сформированных в процессе молекулярно-пучковой эпитаксии с плазменной активацией азота. Спектры фотопроводимости, фотолюминесценции и поглощения демонстрируют сдвиг красной границы межзонных переходов в соответствии с эффектом Бурштейна-Мосса для $n$-InN с различной концентрацией равновесных электронов. Для исследованных образцов наблюдалась абсолютная отрицательная фотопроводимость с наносекундным временем релаксации. Результаты фотоэлектрических, абсорбционных и люминесцентных спектроскопических экспериментов сопоставлены с технологическими параметрами и данными электронной микроскопии.
\end{abstract}

DOI: $10.21883 /$ FTP.2017.12.45169.31

\section{1. Введение}

Интерес к исследованиям оптических и фотоэлектрических свойств эпитаксиальных слоев нитрида индия связан с возможностью реализации на основе этого прямозонного полупроводника с шириной запрещенной зоны $E_{g} \sim 0.65$ эВ [1] активной среды для излучающих и фоточувствительных устройств в ближней ИК области спектра, оптимальной для волоконно-оптических линий связи. InN расширяет рабочий спектральный диапазон для нитридов элементов III группы. Однако трудности технологии получения высококачественных слоев InN существенно тормозят реализацию приборных применений на его основе. Выращиваемые эпитаксиальные слои $\mathrm{InN}$ имеют проводимость $n$-типа, характеризуются большой концентрацией электрически активных примесей $\left(>10^{17} \mathrm{~cm}^{-3}\right)[2]$ и дефектов, что осложняет получение материала с дырочной проводимостью. При всем несовершенстве эпитаксиальных методов выращивания InN во многих лабораториях ведутся работы по приборным применениям InN-структур в качестве светодиодов [3] и детекторов ИК-диапазона [4,5], быстродействующих полевых транзисторов [6]. В эпитаксиальных слоях $n$ - InN наблюдалась эффективная фотолюминесценция [7] и стимулированное излучение при оптической накачке [8]. Однако необходимо отметить, что фотоэлектрические свойства InN остаются исследованными недостаточно. Так, в экспериментах с интенсивным лазерным межзонным возбуждением наблюдался как положительный, так и отрицательный фотоотклик, с характерными длинными, более $10 \mathrm{c}$, временами достижения стационарного состояния [9-11]. Авторы указанных работ связывают отрицательную фотопроводимость с дополнительным рассеянием электронов при захвате фотогенерируемых (неравновесных) дырок на центры рекомбинации. В работе [12] было показано, что медленная отрицательная проводимость возникает вследствие лазерного нагрева $\mathrm{InN}$, но фотоотклик, регистрируемый при слабом возбуждении, показывал времена спада $\sim 1$ с, что не характерно для релаксации фотопроводимости в прямозонном материале. Трудности и противоречия в интерпретации эмиссионных и фотоэлектрических характеристик InN являются следствием сложной дефектной структуры эпитаксиальных слоев, весьма чувствительной к незначительным изменениям параметров ростовых процессов. В данной работе приведены результаты экспериментального определения спектров и кинетики фотопроводимости, фотолюминесценции и поглощения эпитаксиальных слоев InN, выращенных методом молекулярно-пучковой эпитаксии с плазменной активацией азота на подложках сапфира $c-\mathrm{Al}_{2} \mathrm{O}_{3}$ с буферными слоями GaN. Результаты фотоэлектрических, абсорбционных и люминесцентных спектроскопических экспериментов сопоставляются с технологическими параметрами, данными сканирующей электронной микроскопии и рентгеноструктурного анализа.

\section{2. Методика эксперимента}

Рост слоев InN проводился методом молекулярно-пучковой эпитаксии с плазменной активацией азота (МПЭ ПА) на установке STE 3N3 (3АO „НTO“) на 
подложках $c-\mathrm{Al}_{2} \mathrm{O}_{3}$. Слой $\mathrm{InN}$ осаждался на буферный слой, состоящий из слоя AlN толщиной $\sim 0.3$ мкм и слоя $\mathrm{GaN}$ толщиной $\sim 0.9$ мкм. Среднеквадратичная шероховатость поверхности полученных GaN/AlN-буферов была меньше 1 нм, а суммарная плотность дислокаций в них, по данным рентгеноструктурного анализа, составляла $(2-3) \cdot 10^{10} \mathrm{~cm}^{-2}$. Слои $\mathrm{InN}$, выращенные на таких буферных слоях, имели толщину $\leq 1$ мкм.

Для проведения исследований были отобраны структуры со слоями InN, выращенными методом МПЭ ПА тремя различными способами, широко используемыми в литературе. Образец InN 35 выращен „традиционным“ методом: первые $\sim 25 \mathrm{Hм}$ слоя InN осаждались при пониженной температуре $\sim 430^{\circ} \mathrm{C}$, а затем температура роста поднималась до $\sim 480^{\circ} \mathrm{C}$. Из литературы хорошо известно, что осаждение на начальных этапах зародышевого слоя при низкой температуре способствует улучшению кристаллического качества пленки $\operatorname{InN}[13,14]$. Рост пленки $\mathrm{InN}$ проходил в азотобогащенных условиях при соотношении потоков элементов III и V групп $\sim 0.8$, при этом поток индия на подложку составлял $\sim 0.4$ мкм/ч. В образцах 55 и 61 для роста пленок InN применялся метод эпитаксии с модуляцией потока металла (metal-modulated epitaxy, MME), суть которого состоит в попеременном росте в индийобогащенных условиях роста и выдержке поверхности роста под потоком азота $[15,16]$. Во время подачи потока In на подложку возникают металлобогащенные условия роста, способствующие поверхностной диффузии адатомов. Последующая выдержка поверхности роста в потоке азота приводит к формированию слоя $\mathrm{InN}$ из излишков атомов In, накопившихся во время первой части периода осаждения. Далее этот процесс многократно повторяется. В образцах 55 и 61 цикл составлял 30 с, из которых 15 с проходил рост в металлобогащенных условиях, а 15 с поверхность роста выдерживалась в потоке азота. Во время фазы роста в металлобогащенных условиях на поверхности скапливалось 1.5-2 монослоя (МС) металлического In. Согласно теории [17], при росте нитридов в металлобогащенных условиях поверхность роста смачивается 2 МС металла, а остальной излишек металла собирается в капли. Ограничение в накоплении 1.5-2 монослоя In во время фазы роста в металлобогащенных условиях необходимо для подавления образования капель металлического индия на поверхности и, таким образом, проблем с формированием кластеров металлического In внутри слоя InN $[18,19]$. Средняя скорость роста слоев InN в структурах 55 и 61 составляла $\sim 0.25$ мкм/ч. Отличие образцов состояло в том, что в образце 61 осаждение $\operatorname{InN}$ начиналось при низкой температуре (как в образце 35). Первые 5 мин ( $\sim 20$ нм по толщине) рост InN в образце 61 проходил при температуре $\sim 430^{\circ} \mathrm{C}$, а затем температура увеличивалась до $\sim 450^{\circ} \mathrm{C}$. Слой $\mathrm{InN}$ в образце 55 выращен при фиксированной температуре $\sim 450^{\circ} \mathrm{C}$.

Выращенные структуры исследовались методами сканирующей электронной микроскопии (СЭМ) на микроскопе Carl Zeiss Supra 50VP и рентгеноструктурного анализа на дифрактометре Bruker D8 Discover. Спектры поглощения записывались на фурье-спектрометре с быстрым сканированием IFS 125HR „BRUKER“ с вольфрамовой лампой накаливания в качестве источника излучения в диапазоне 0.4-1.9 эВ и инфракрасным термоэлектрическим фотоприемником с окном из $\mathrm{KBr}$. Для возбуждения фотоотклика с временны́м разрешением использовался импульсный оптический параметрический генератор OPO („Solar $\mathrm{LS}^{\text {“ }}$ ), перестраиваемый в диапазоне $\lambda=0.44-18$ мкм, с длительностью импульсов $\leq 10$ нс. Для регистрации фотоотклика использовалась стандартная схема включения фотосопротивления с подачей постоянного смещения на образец через согласованное сопротивление нагрузки, сигнал с которого измерялся с помощью осциллографа WaveSurfer-432 („LeCroy“). Контакты к слою InN приготавливались пайкой индием. Сопротивление образцов не превышало 200 Ом и не изменялось заметно при понижении температуры от комнатной до $4.2 \mathrm{~K}$. Предельное временно́е разрешение $(\sim 10 \mathrm{Hc})$ при регистрации фотопроводимости определялось длительностью импульсов ОРО. Спектры стационарной фотолюминесценции (ФЛ) исследовались при температурах 77 и $300 \mathrm{~K}$. Для возбуждения ФЛ использовалось непрерывное лазерное излучение с длиной волны 440 нм и мощностью до $100 \mathrm{MB}$. Регистрация спектров ФЛ в диапазоне $1-2.2$ мкм осуществлялась с помощью решеточного монохроматора Acton 2300і и многоканального фотоприемника OMA-V:1024-2.2 (Princeton Instruments) на основе линейки фотодиодов InGaAs.

\section{3. Экспериментальные результаты и их обсуждение}

Для всех исследованных образцов были зарегистрированы спектры фотопроводимости (РС), фотолюминесценции (PL) и поглощения (Abs). На рис. 1 сопоставлены все три спектра фотовозбуждения для образца InN 35, а также спектры поглощения вблизи края зоны для образцов 55 и 61. Наибольший интерес представляют спектр и кинетика фотопроводимости, поскольку в предшествующих работах наблюдался фотоотклик только при интенсивном межзонном возбуждении с фиксированной энергией кванта, существенно превышающей ширину запрещенной зоны [9-12]. Поскольку спектр фотопроводимости регистрировался при импульсном возбуждении, можно утверждать, что наблюдаемый фотоотклик не связан с эффектом лазерного нагрева образца [12] и с медленным захватом генерируемых дырок на центры рекомбинации [10], т. е. с механизмами формирования фотоотклика $\mathrm{InN}$, которые предлагались ранее для интерпретации медленного отрицательного фотоотклика InN.

Основной особенностью фотоотклика при комнатной температуре для исследованных в данной работе образцов является уменьшение проводимости при межзонном 


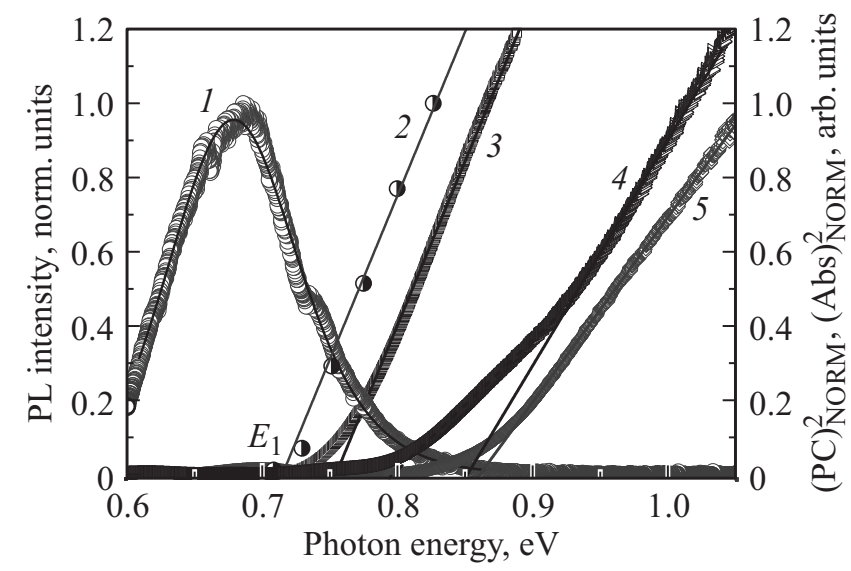

Рис. 1. Нормированные спектры PL (1), (PC) ${ }^{2}(2)$ и $(\mathrm{Abs})^{2}(3)$ образца InN 35, $(\mathrm{Abs})^{2}$ (4) образца $\mathrm{InN} 55$ и $(\mathrm{Abs})^{2}(5)$ образца InN 61. Сплошные линии на спектрах $(\mathrm{PL})^{2}$ и $(\mathrm{Abs})^{2}-$ результаты линейной аппроксимации. Стрелкой указана величина $E_{1}=E_{g}^{*}+E_{\mathrm{F}}$, определенная при описании спектра фотолюминесценции. Сплошная линия для спектра фотолюминесценции - описание с помощью выражения (1).

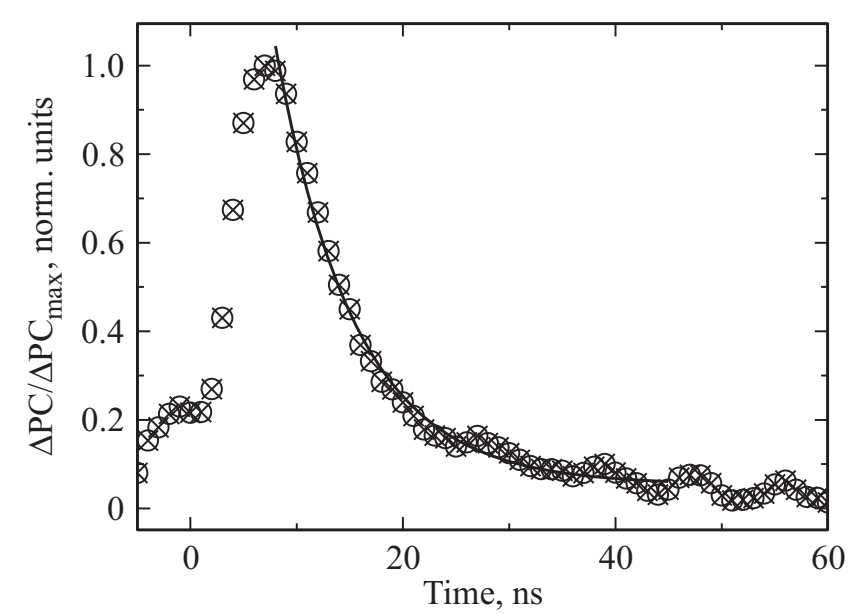

Рис. 2. Кинетика отрицательной фотопроводимости образца InN 35 при возбуждении с энергией кванта 800 мэВ при температуре $294 \mathrm{~K}$. Сигнал нормирован на максимальное отрицательное изменение РС.

освещении - абсолютная отрицательная фотопроводимость. Кинетика фотоотклика на возбуждение наносекундным импульсом, приведенная на рис. 2, показывает, что отрицательный фотоотклик релаксирует за время $\approx 8$ нс, которое близко к временно́му разрешению системы регистрации и является оценкой верхней границы для времени релаксации фотопроводимости. Наблюдаемое в данной работе время релаксации отрицательной фотопроводимости на несколько порядков меньше времени спада отрицательной фотопроводимости InN, обнаруженной ранее в образцах с близкими электрофизическими характеристиками. Можно предположить, следуя $[9,10]$, что наносекундный отрицательный фотоотклик связан с уменьшением подвижности электронов, превышающим увеличение проводимости вследствие генерации неравновесных носителей при межзонном освещении.

Приведенные на рис. 1 нормированные зависимости квадратов сигнала фотопроводимости и поглощения света от энергии фотонов близки и хорошо описываются, исключая области хвостов состояний, линейными функциями, экстраполяция которых к $(\mathrm{Abs})^{2}=0$ и $(\mathrm{PC})^{2}=0$ дает значения края поглощения для межзонных переходов $E_{1}$. Для слоя $\mathrm{InN}$ образца 35 край поглощения равен $E_{1}=715$ и 754 мэВ для фотопроводимости и поглощения соответственно и $E_{1}=860$ мэВ для поглощения в InN образца 61. Два линейных участка в спектре поглощения образца 55 дают значения $E_{1}=800$ и 850 мэВ. Линейная зависимость квадрата коэффициента поглощения и квадрата сигнала фотоотклика от энергии фотона свидетельствует о прямых разрешенных переходах в InN при оптическом возбуждении.

Положение „красной“ границы поглощения и фотопроводимости в исследованных образцах определяется эффектом Бурштейна-Мосса в вырожденном прямозонном полупроводнике для образцов с различной концентрацией равновесных электронов. В доказательство этого утверждения можно привести результаты анализа спектров фотолюминесценции, которые хорошо описываются в рамках модели эмиссии в прямозонном вырожденном полупроводнике [20]:

$$
\begin{aligned}
I_{\mathrm{PL}}(\hbar \omega) & \propto\left[\left(\hbar \omega-E_{g}^{*}\right) / E_{g}^{*}\right]^{\gamma / 2} / \\
& \left(1+\exp \left\{\left(\frac{\mu}{m_{e}}\left(\hbar \omega-E_{g}^{*}\right)-E_{\mathrm{F}}\right) / T^{*}\right\}\right),
\end{aligned}
$$

где $E_{g}^{*}$ - ширина запрещенной зоны, зависящая от концентрации свободных носителей и температуры, $\mu=m_{e} m_{h} /\left(m_{e}+m_{h}\right)$ - приведенная масса электрона и дырки, $E_{\mathrm{F}}$ - энергия Ферми электронов, $T^{*}-$ эффективная температура, а параметр $\gamma$ определяется типом переходов. Аппроксимация спектра фотолюминесценции с помощью выражения (1) позволяет оценить величину $E_{1}=E_{g}^{*}+E_{\mathrm{F}}$, соответствующую краю поглощения в InN. Так, для образца 35 при комнатной температуре (см. спектр 1 на рис. 1) $E_{1}=680$ мэВ.

На рис. 3 приведены спектры стационарной фотолюминесценции исследованных слоев при $T=78 \mathrm{~K}$. Спектры нормированы на максимальное значение, поскольку интенсивность ФЛ, зависящую от большого числа параметров, достаточно трудно использовать в количественных определениях. Высокоэнергетический край спектров фотолюминесценции не связан с генерацией неравновесных носителей при использованных низких уровнях накачки. Для описания спектров достаточно учета эффекта Бурштейна-Мосса в модели (1), в этом случае изменения $E_{1}=E_{g}^{*}+E_{\mathrm{F}}$ согласуются с увеличением концентрации равновесных электронов, в ряду образцов $35,55,61$.

Используя известную зависимость края собственного поглощения $\mathrm{InN}\left(E_{1}\right)$ от концентрации носителей заря- 


\begin{tabular}{c|c|c|c|l}
\hline \multirow{2}{*}{ Образцы } & \multicolumn{4}{|c}{ Концентрация электронов, $n \cdot 10^{18} \mathrm{~cm}^{-3}$} \\
\cline { 2 - 5 } & Эффект Холла & $\mathrm{Abs}$ & $\mathrm{PC}$ & $\mathrm{PL}$ \\
\hline 35 & 5 & 5 & 3 & 1.3 \\
55 & 13 & $15 / 9$ & Не определена & 3 \\
61 & 17 & 17 & $\gg \gg$ & 10
\end{tabular}

да [15], были определены концентрации электронов в исследуемых образцах (см. таблицу) по спектрам фотолюминесценции, поглощения света и фотопроводимости. Результаты, полученные на основе спектров поглощения света, хорошо согласуются с данными, полученными из измерений эффекта Холла в исследуемых образцах.

Сопоставим наблюдаемые особенности в спектрах фотовозбуждения $\mathrm{InN}$, различия края межзонного поглощения $E_{1}$ в спектрах поглощения, фотолюминесценции и фотопроводимости с ростовыми параметрами и возникающими особенностями структур. Результаты, представленные на рис. 1,3 и в таблице, показывают увеличение концентрации свободных носителей и соответствующий сдвиг уровня Ферми при переходе к эпитаксии InN методом MME в образцах 55 и 61. Результаты рентгенодифракционных измерений полуширины отражений в направлении (0004) и $(10-12)$ для исследованных образцов оказались близки: $\sim 0.25^{\circ}$ (образцы 35 и 61 ) и $\sim 0.22^{\circ}$ (образцы 55) для отражения (0004) и $\sim 0.5^{\circ}$ (образцы 35 и 61 ) и $\sim 0.48^{\circ}$ (образец 55) для отражения (10-12), при значительных отличиях в структуре слоев InN, обнаруженных методам СЭМ. Из данных по полуширинам кривых качания рассчитывались значения плотности винтовых и краевых прорастающих дислокаций по модели из работы [21]. Плотность винтовых дислокаций составила $\sim 1.8 \cdot 10^{9} \mathrm{~cm}^{-2}$ (образцы 35 и 61) и $\sim 1.4 \cdot 10^{9} \mathrm{~cm}^{-2}$ (образец 55), плотность краевых дислокаций составила $\sim 3.4 \cdot 10^{10} \mathrm{~cm}^{-2}$ (образцы 35 и 61) и $\sim 3.2 \cdot 10^{10} \mathrm{~cm}^{-2}$ (образец 55). По-видимому, увеличение концентрации электронов определяется особенностями образования электрически активных примесно-дефектных центров в процессе MEЕ, не связанных с дислокациями.

Рост величины $E_{1}=E_{g}^{*}+E_{\mathrm{F}}$ при определении ее в ряду спектров поглощения, фотолюминесценции и фотопроводимости (см. рис. 1) связывается с неоднородностью слоев $\mathrm{InN}$, содержащих области с различной концентрацией дефектов. Вклад областей с минимальной концентрацией дефектов доминирует в сигнале фотолюминесценции, поскольку безызлучательная релаксация (например, по механизму Шокли-Рида-Холла, при наличии примесных уровней в запрещенной зоне) уменьшает долю сигнала фотолюминесценции от менее совершенных участков слоя InN. Сигнал фотопроводимости определяется отношением числа генерируемых носителей к числу равновесных и пропорционален подвижности носителей заряда. Оба фактора увеличивают вклад более качественных, „чистых“ областей в фотоотклик. Одностадийный процесс поглощения фотонов уравнивает вклады различных областей, поэтому измерения поглощения дают максимальные значения энергии Ферми и концентрации свободных носителей соответственно. Косвенным подтверждением изложенного предположения являются изображения, полученные методом сканирующей электронной микроскопии. В образце 35 , выращенном „традиционным“ методом, при соотношении потоков III/V 0.8 значительную часть $\mathrm{InN}$ составляет неоднородный нанопористый слой, постепенно превращающийся в сплошной (см. рис. 4). Возможными причинами такой структуры слоя InN могут быть как постоянные азотобогащенные условия, так и достаточно высокие температуры роста $\left(\sim 480^{\circ} \mathrm{C}\right)$, способствующие трехмерному росту пленки.

Неоднородность исследованных слоев не может быть отнесена к образованию областей гексагональной и кубической фаз InN, как это сделано в [22], где наблюдались изменения красной границы в спектрах поглощения, подобные наблюдаемым в спектре образца InN 55 (кривая 4 на рис. 1), поскольку рентгеноструктурные

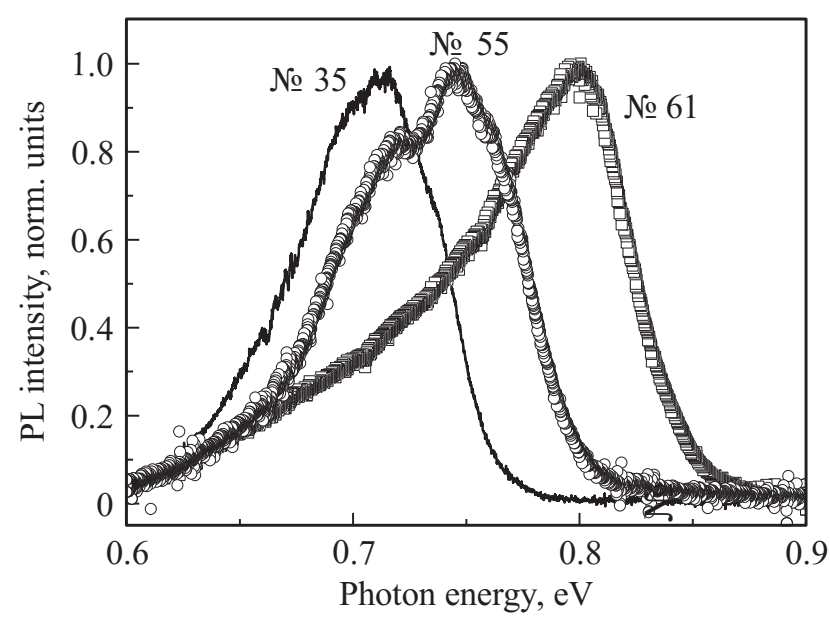

Рис. 3. Спектры фотолюминесценции эпитаксиальных слоев $n$-InN, выращенных методом МПЭ ПА, с различной концентрацией свободных носителей. Спектры измерены при $T=78 \mathrm{~K}$.

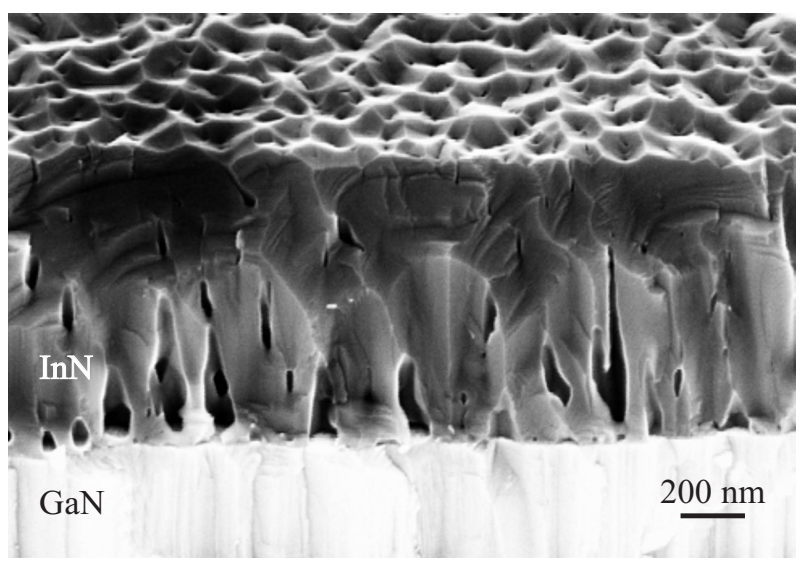

Рис. 4. Снимок поперечного скола образца 35 с эпитаксиальным слоем InN, полученный с помощью СЭМ. 
данные свидетельствуют о наличии только гексагональной фазы InN в исследованных слоях.

\section{4. Заключение}

В работе представлены результаты исследований спектров фотопроводимости, фотолюминесценции, фотовозбуждения и поглощения света эпитаксиальных слоев InN c концентрацией свободных носителей $10^{18}-10^{19} \mathrm{~cm}^{-3}$, сформированных в процессе молекулярно-пучковой эпитаксии с плазменной активацией азота. Спектры фотопроводимости, фотолюминесценции и поглощения демонстрируют сдвиг красной границы межзонных переходов в соответствии с эффектом Бурштейна-Мосса для $n$-InN с различной концентрацией равновесных электронов, зависящей от ростовых параметров. Концентрации равновесных электронов в исследованных образцах не зависят от плотности винтовых и краевых дислокаций. При импульсном возбуждении в диапазоне $0.6-1$ эВ в образцах с концентрацией электронов $>10^{18} \mathrm{~cm}^{-3}$ наблюдалась абсолютная отрицательная межзонная фотопроводимость с наносекундным временем релаксации при комнатной температуре.

Работа выполнена при финансовой поддержке РФФИ (грант № 16-29-03374) и программ РАН. В работе использовано оборудование ЦКП ИФМ РАН „Физика и технология микро- и наноструктур“.

\section{Список литературы}

[1] V.Yu. Davydov, A.A. Klochikhin, V.V. Emtsev, A.V. Sakharov, S.V. Ivanov, V.A. Vekshin, F. Bechstedt, J. Furthmueller, J. Aderhold, J. Graul, A.V. Mudryi, H. Harima, A. Hashimoto, A. Yamamoto, J. Wu, H. Feick, E.E. Haller. Phys. Status Solidi B, 234 (3), 787 (2002).

[2] Z. Mi, S. Zhao. Phys. Status Solidi B, 252 (5), 1050 (2015).

[3] G.G. Wu, W.C. Li, C.S. Shen, F.B. Gao, H.W. Liang, H. Wang, L.J. Song, G.T. Du. Appl. Phys. Lett., 100, 103504 (2012).

[4] L.H. Hsu, C.T. Kuo, J.K. Huang, S.C. Hsu, H.Y. Lee, H.C. Kuo, P.T. Lee, Y.L. Tsai, Y.C. Hwang, C.F. Su, J.H. He, S.Y. Lin, Y.J. Cheng, C.C. Lin. Opt. Express, 23 (24) 31150 (2015).

[5] A. Shetty, M. Kumar, B. Roul, K.J. Vinoy, S.B. Krupanidhi. J. Nanosci. Nanotechn., 16 (1), 709 (2016).

[6] M. Oseki, K. Okubo, A. Kobayashi, J. Ohta, H. Fujioka. Scientific Reports, 4, 3951 (2014).

[7] A.A. Klochikhin, V.Y. Davydov, V.V. Emtsev, A.V. Sakharov, V.A. Kapitonov, B.A. Andreev, H. Lu, W.J. Schaff. Phys. Status Solidi B, 242 (4), R33 (2005).

[8] M.S. Hu, G.M. Hsu, K.H. Chen, C.J. Yu, H.C. Hsu, L.C. Chen, J.S. Hwang, L.S. Hong, Y.F. Chen. Appl. Phys. Lett., 90, 123109 (2007).

[9] P.C. Wei, S. Chattopadhyay, M.D. Yang, S.C. Tong, J.L. Shen, C.Y. Lu, H.C. Shih, L.C. Chen, K.H. Chen. Phys. Rev. B, 81 (4), 045306 (2010).

[10] L. Guo, X. Wang, L. Feng, X. Zheng, G. Chen, X. Yang, F. Xu, N. Tang, L. Lu, W. Ge, B. Shen. Appl. Phys. Lett., 102, 072103 (2013).
[11] L. Guo, X.Q. Wang, X.T. Zheng, X.L. Yang, F.J. Xu, N. Tang, L.W. Lu, W.K. Ge, B. Shen, L.H. Dmowski, T. Suski. Scientific Reports, 4, 4371 (2014).

[12] T.T. Kang, Y. Zhang, P.P. Chen, Z.H. Wang, A. Yamamoto. Appl. Phys. Lett., 110 (4), 042104 (2017).

[13] S.V. Ivanov, T.V. Shubina, V.N. Jmerik, V.A. Vekshin, P.S. Kop'ev, B. Monemar. J. Cryst. Growth, 269, 1 (2004).

[14] H. Xiao, X. Wang, J. Wang, N. Zhang, H. Liu, Y. Zeng, J. Li, Z. Wang. J. Cryst. Growth, 276, 401 (2005).

[15] J. Wu, W. Walukiewicz, S.X. Li, R. Armitage, J.C. Ho, E.R. Weber, E.E. Haller, H. Lu, W.J. Schaff, A. Barcz, R. Jakiela. Appl. Phys. Lett., 84, 2805 (2004).

[16] N. Miller, E.E. Haller, G. Koblmüller, C. Gallinat, J.S. Speck, W.J. Schaff, M.E. Hawkridge, K.M. Yu, J.W. Ager. Phys. Rev. B, 84, 075315 (2011).

[17] M. Moseley, D. Billingsley, W. Henderson, E. Trybus, W.A. Doolittlea. J. Appl. Phys., 106, 014905 (2009).

[18] S.V. Ivanov, T.V. Shubina, T.A. Komissarova, V.N. Jmerik. J. Cryst. Growth, 403, 83 (2014).

[19] T.P. Bartel, C. Kisielowski, P. Specht, T.V. Shubina, V.N. Jmerik, S.V. Ivanov. Appl. Phys. Lett., 91, 101908 (2007).

[20] В.Ю. Давыдов, А.А. Клочихин. ФТП, 38, 897 (2004).

[21] M.A. Moram, M.E. Vickers. Rep. Prog. Phys., 72, 036502 (2009).

[22] Y. Zhang, T. Kimura, K. Prasertusk, T. Iwabuchi, S. Kumar, Y. Liu, R. Katayama, T. Matsuoka. Thin Sol. Films, 536, 152 (2013).

Редактор Г.А. Оганесян

\section{The peculiarities of photoexcitation spectra of InN epitaxial layers grown by the PAMBE technique}

\author{
P.A. Bushuykin ${ }^{1}$, A.V. Novikov ${ }^{\mathbf{1}, 2}$, B.A. Andreev ${ }^{\mathbf{1 , 2}}$, \\ D.N. Lobanov ${ }^{1,2}$, P.A. Yunin ${ }^{1}$, E.V. Skorokhodov ${ }^{1}$, \\ L.V. Krasil'nikova ${ }^{1,2}$, E.V. Demidov' ${ }^{1}$, G.M. Savchenko ${ }^{3}$, \\ V.Yu. Davydov ${ }^{3}$ \\ ${ }^{1}$ Institute for Physics of Microstructures, \\ Russian Academy of Sciences, \\ 603950 Nizhny Novgorod, Russia \\ ${ }^{2}$ Lobachevsky State University of Nizhny Novgorod, \\ 603950, Nizhny Novgorod, Russia \\ ${ }^{3}$ loffe Institute, \\ 194021 St. Petersburg, Russia
}

\begin{abstract}
In this paper, the results of the investigation of the photoexcitation spectra of $\mathrm{InN}$ epilayers with free electron concentration $10^{18}-10^{19} \mathrm{~cm}^{-3}$ formed in the process of molecularbeam epitaxy with plasma activation of nitrogen are presented. The photoconductivity, photoluminescence and absorption spectra show shift of the red edge of the interband transitions in accordance with Burshtein-Moss effect for $n$-InN with a different concentration of equilibrium electron. Absolute negative photoconductivity with nanosecond relaxation time was observed for the studied samples. The results of photoelectric, absorption and luminescence spectroscopic experiments were compared with technological parameters and electron microscopy data.
\end{abstract}

